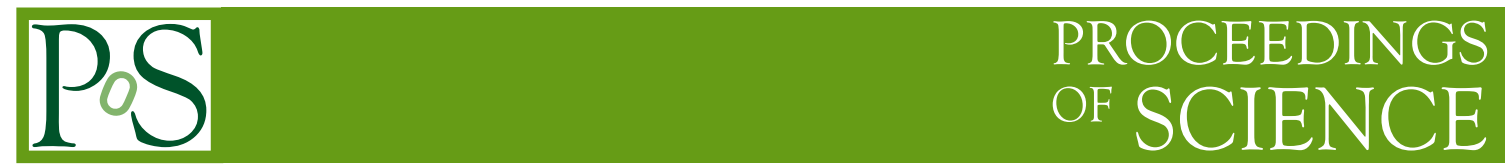

\title{
Gamma Rays: Direct Observations
}

\section{Riccardo Rando*}

University of Padova, Italy and INFN Sezione di Padova, Italy

E-mail: riccardo.rando@pd.infn.it

These proceedings give a summary of the Gamma Ray Direct parallel session at the 36th International Cosmic Ray Conference, held in Madison, WI, USA. Approximately 63 contributions were submitted, detailing the advancement in observation and modeling of a wide range of sources.

36th International Cosmic Ray Conference -ICRC2019-

July 24th - August 1st, 2019

Madison, WI, U.S.A.

${ }^{*}$ Speaker. 


\section{Introduction}

There were 23 oral presentations in the Gamma ray direct parallel session, in addition to about 40 submitted poster contributions. This is an overwhelming amount of information to summarize while trying to keep a readable, logical structure. I will work under the assumption that this is an introductory guide to the material available elsewhere, and I will be favoring contributions with proceedings submitted to $\operatorname{PoS}^{1}$. I will only reference the latter, please refer to the cited contributions for the complete references.

I will structure the report in sections, listing the topics into broad classes of sources roughly as a function of their distance from the observer. I will not follow this scheme too strictly within each section, though.

As a final word of warning, this report is filtered by my personal views, with the unavoidable slips and misunderstandings for which the original authors should not be blamed. I will summarize my impression of this parallel session, hoping that the reader will find a confirmation in the following sections. We are in a very fruitful era:

- we have several, mature instruments operating at all times, providing us with precise and accurate measurements in the $\gamma$-ray portion of the electromagnetic spectrum;

- a wide array of excellent instruments monitor most of the other regions of the electromagnetic spectrum and several experiments collect many other messengers (cosmic rays, neutrinos, gravitational waves, ...);

- the quality of the available data is putting the existing models under some duress and in several cases the models will need major updates to properly describe the measurements.

Nonetheless some words of dissatisfaction are voiced over and over.

- We deal often with limited statistics, especially in the energy range above $\sim 10 \mathrm{GeV}$. On the other hand, in this photon-starved regime statistics is improving linearly with time.

- Many features, at odds with the current models, are right at the boundary between statistical flukes and full significance.

- A few portions of the electromagnetic spectrum are not monitored at all, and as a consequence many competing models are available which differ significantly in the blind regions.

\section{Solar system}

The $\gamma$-ray flux from the quiescent Sun is the sum of the emission from the solar limb due to cosmic ray cascades in the solar atmosphere, and of the inverse Compton scattering of cosmic-ray electrons on solar photons in the heliosphere. The measurement in [1], making use of 9 years of Fermi-LAT data, shows a clear temporal modulation in the $1-10 \mathrm{GeV}$ range, consistent with expectations from the solar cycle: the high flux state corresponds to the solar minimum around year

\footnotetext{
${ }^{1}$ https://pos.sissa.it/358
} 
2009. Less obvious is the apparent modulation at high energies: of 7 events with $E>100 \mathrm{GeV}, 6$ were detected from August 2008 to January 2010 (last solar minimum), then nothing for 7.8 years, and finally one new event in February 2018 at the onset of the current solar minimum. Remarkably, the solar flux estimated for the last minimum is higher the recent HAWK upper limits, indicating that we could be able to observe the Sun up to TeV energies. The authors report a hint of spatial disuniformity, with events at $E>50 \mathrm{GeV}$ appearing to cluster on a band lying at the solar equator. The above spectral and spatial features are to be confirmed by the data that will be collected during the current solar minimum.

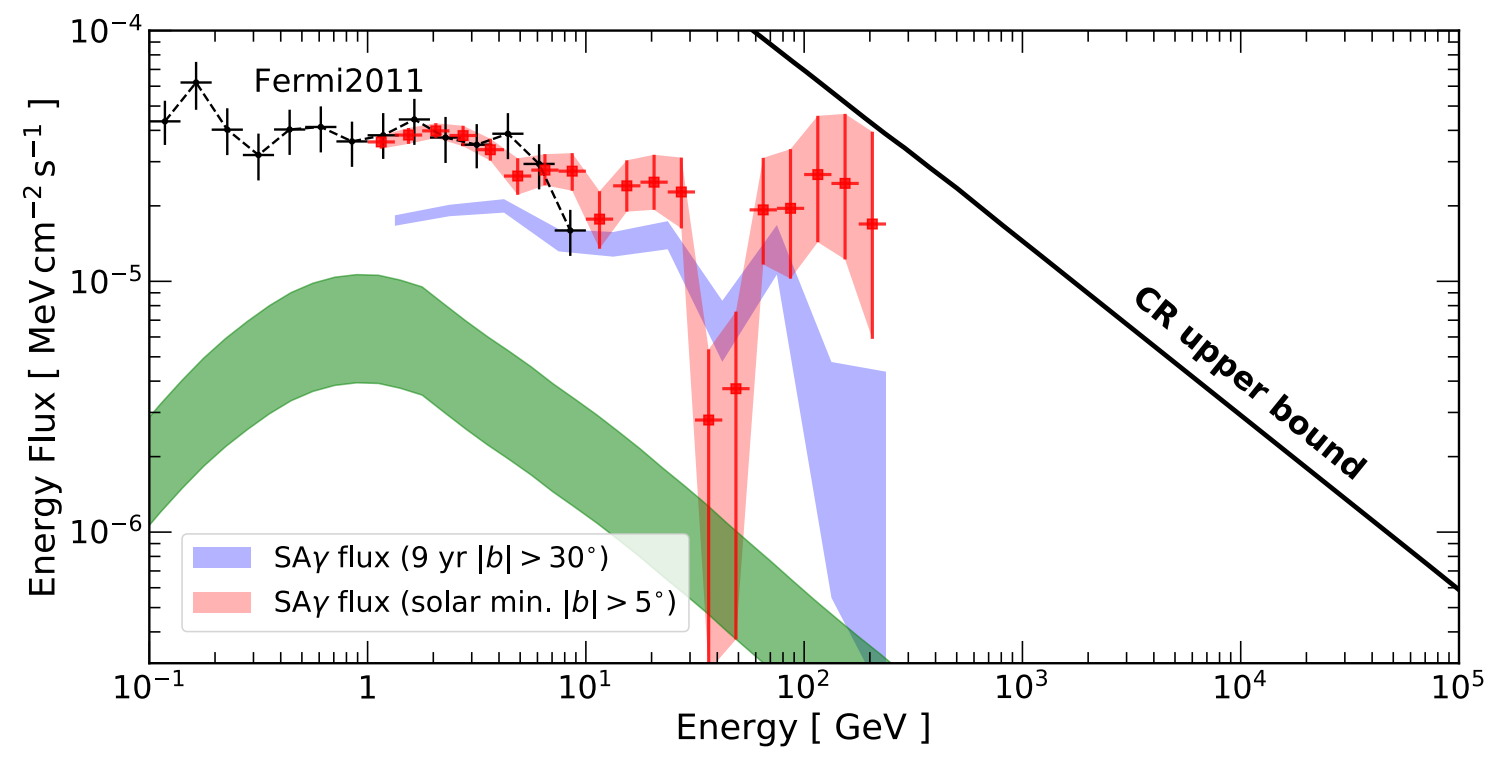

Figure 1: Spectrum for the solar emission of cosmic-ray origin. Black points: Fermi-LAT analysis, last solar minimum; blue band: analysis in [1], 10 years of data; red: same, last solar minimum only; green: theoretical model.

\section{Galactic sources}

Local pulsars are among the best candidates to explain the positron excess above $\sim 10 \mathrm{GeV}$ reported e.g. by AMS-02 and PAMELA . On this topic, the recent detection of extended emission around Geminga and Monogem by MILAGRO and HAWK seems to indicate evidence of $\mathrm{e}^{ \pm}$diffusing away from the pulsar and causing inverse Compton upscattering of the microwave background photons. In [2] the authors report the detection of a diffuse halo around Geminga in Fermi-LAT data and a negative result for Monogem. This allows to derive the $\mathrm{e}^{+}$flux at Earth under the assumption of a two-zone diffusion model, with an inhibited diffusion region around the pulsars corresponding to the angular size of the halo of Geminga. The maximum contribution to the local $\mathrm{e}^{+}$flux amounts to $10 \%$ (at $800 \mathrm{GeV}$ ) for Geminga, and to an upper limit of $3 \%$ for Monogem. Based on these figures, the population of Galactic Pulsar Wind Nebulae (PWNs) remains a viable candidate to explain the estimated $\mathrm{e}^{+}$emission. In this work two different spatial templates for the halo of Geminga are tested: one including proper motion of the pulsar and one not. The former is preferred at $4 \sigma$, providing the first direct detection of proper motion in $\gamma$-ray astronomy. 
AR Scorpii is a nearby ( $\sim 100 \mathrm{pc})$ white dwarf binary system, with a spectrum dominated by non-thermal emission from radio to $X$ frequencies. In [3] the authors look for $\gamma$-ray emission in 10 years of Fermi-LAT data, finding a $\sim 3.9 \sigma$ excess between $100 \mathrm{MeV}$ and $500 \mathrm{GeV}$. Notably, this is a fairly complex region (including the Rho Ophiuchi molecular cloud) and further analysis is warranted.

Eta Carinae is a stellar system consisting of two bright stars (luminosity in the order of $10^{6}$ $\left.\mathrm{L}_{\odot}\right)$. The stars rotate around one another, with the interacting winds accelerating particles and producing $\gamma$-ray and X-ray emission which in turn interacts with the intense thermal photon flux. In [4] the authors analyze the available multi-wavelength data (UV, X, $\gamma$ ), to characterize the spectrum and orbital variability. In particular, the role of future observation in the Cherenkov band (by the Cherenkov Telescope Array) is discussed, to clarify the geometry of the system and the physics of the shocks at a high level of detail. The value of an instrument sensitive in the $\mathrm{MeV}$ range is also discussed, in order to discriminate between lepto-hadronic and hadronic emission models.

HESS J1825-137 is the largest known PWN, and one of the most luminous. In [5] the authors analyze 10 years of Fermi-LAT data, performing a morphological and spectral analysis, confirming the previous HESS results at higher energies. The observed increase of apparent size towards lower energies in the $\mathrm{GeV}$ band is unexpected, since the age of the PWN is smaller than the energy losses in a simple diffusion-energy loss scenario.
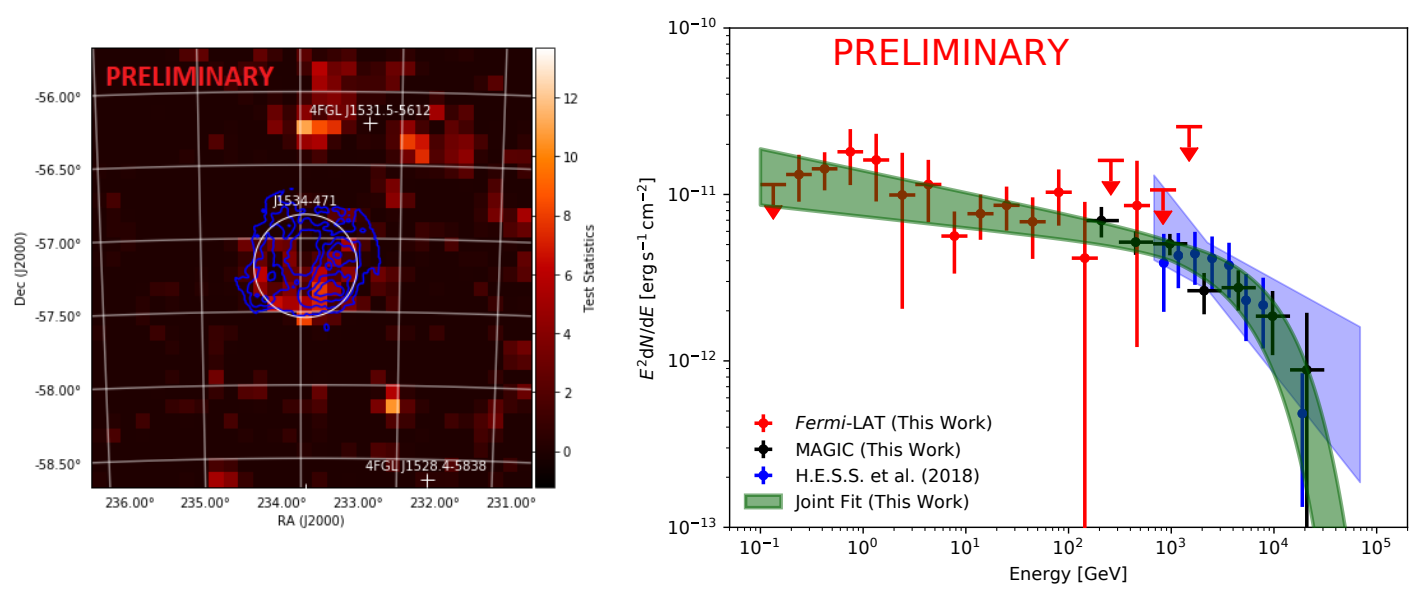

Figure 2: Left: morphology of J1534-571, excess counts in Fermi-LAT data compared to the extension map from HESS; from [6]. Right: spectral energy distribution of J1912+101, with combined fit of Fermi-LAT and MAGIC data; from [7].

Several other HESS candidate Supernova remnants (SNR) are analyzed in different contributions, in terms of morphology and spectrum using 10 years of Fermi-LAT data. In [6] J1614-518 is found to host a pulsar, first identified as a point source with the characteristic curved spectrum, then confirmed by a blind search for pulsation (no counterparts at other wavelengths are known). This suggests that the emission is not related to the SNR but to a PWN. A morphological and spectral study of J1534-571 is performed: the source is clearly extended, with a simple power law spectrum. It is worth pointing out how limited the available statistics is: about 100 photons are attributed to J1534-571, roughly one per month. The combined analysis of Fermi-LAT and 
MAGIC data for J1912+101 in [7] reveals a continuous spectrum with a cutoff at $\sim 9.6 \mathrm{TeV}$ and consistent morphology, typical of a SNR and opposed to the two-component spectrum of a PWN (from pulsar and nebula). J1641-463 is an example of the opposite behavior [8]: no extension is observed, at $\mathrm{GeV}$ energies a clear curvature is detected in the spectrum suggesting a pulsar, while a hard power-law spectrum at $\mathrm{TeV}$ energies is reported by HESS. The search for pulsation on the pulsar candidate is ongoing.

Supernova remnants often evolve in material with high abundance of heavy elements, such as carbon or oxygen. In [9] the authors simulate the interactions of cosmic rays with the surrounding material for different realizations of a SNR in a given evolutionary state, varying the composition of the cosmic rays and of the ambient gas. The results indicate that the $\gamma$-ray spectrum below $3 \mathrm{GeV}$ varies depending on composition, making it difficult to search for non-thermal bremsstrahlung, and that the high-energy cut-off moves at lower energy for a heavy composition, so that fewer $\gamma$ rays are expected from SNRs evolving in a zone with heavy composition.

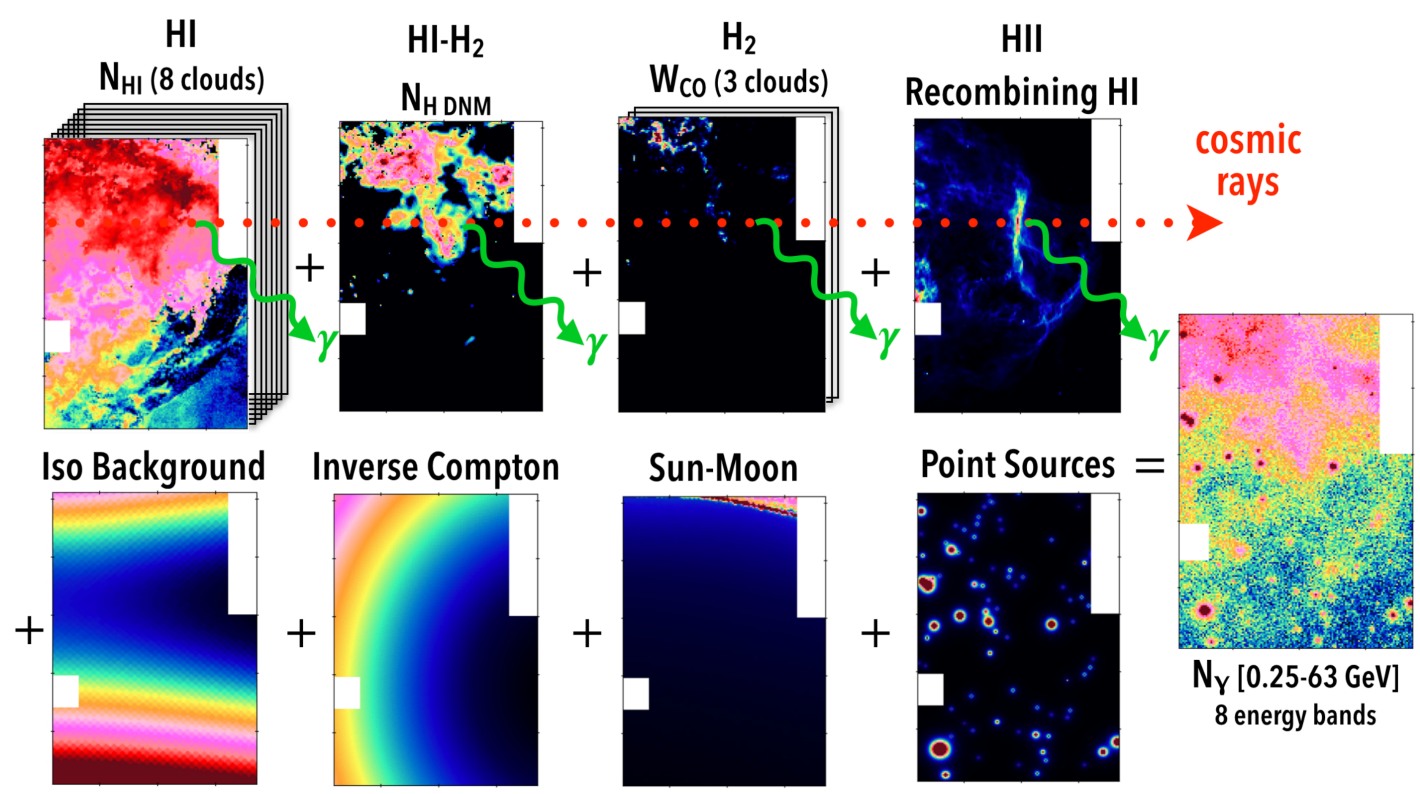

Figure 3: Modeling the interstellar diffuse emission with emission templates in the region around the OrionEridanus super-bubble; from [10].

For structures much larger than these the analysis of Fermi-LAT data requires a in-depth study of the interstellar emission background. The Orion-Eridanus super-bubble is the closest site of active high-mass star formation, reaching as close as $\sim 100 \mathrm{pc}$. Winds from massive stars and supernovae at a rate of about one per Myr over the past $12 \mathrm{Myr}$ created a massive cavity in the interstellar medium (roughly 150 by $300 \mathrm{pc}$ ) in the approximate direction of the Galactic anticentrum. Dealing with an angular size $50^{\circ}$ by $50^{\circ}$, in [10] the authors proceed to model the interstellar emission along that line of sight; the complexity of the model can be estimated by Fig. 3. The global $\gamma$-ray emissivity spectrum thus obtained is consistent with the average for the local medium, with no indication of in-situ acceleration or re-acceleration of cosmic rays, or any effect of the turbulent gas on the propagation of cosmic rays in the bubble. This result contrasts with what was 
observed for the Cygnus-X super-bubble, where a cocoon of freshly accelerated cosmic rays has been detected.

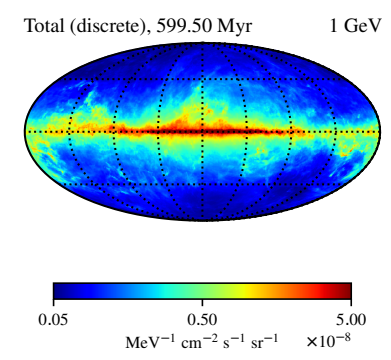

SA50, $599.50 \mathrm{Myr} \quad 1 \mathrm{GeV}$
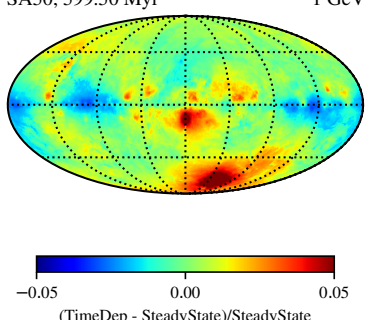
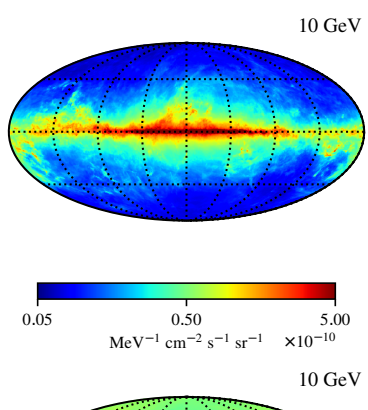

$10 \mathrm{GeV}$
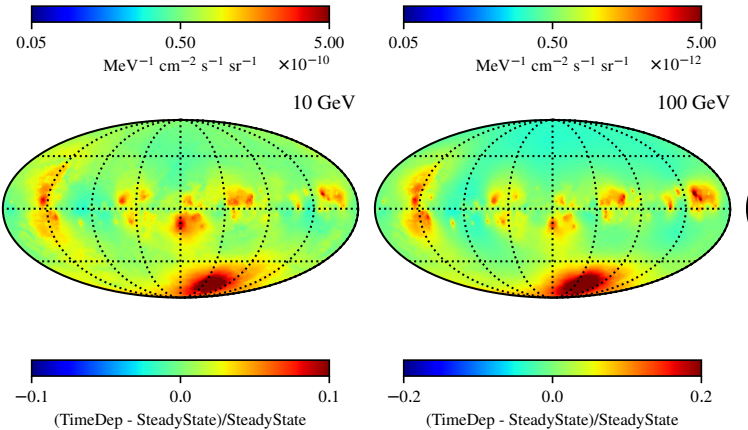

$1 \mathrm{TeV}$
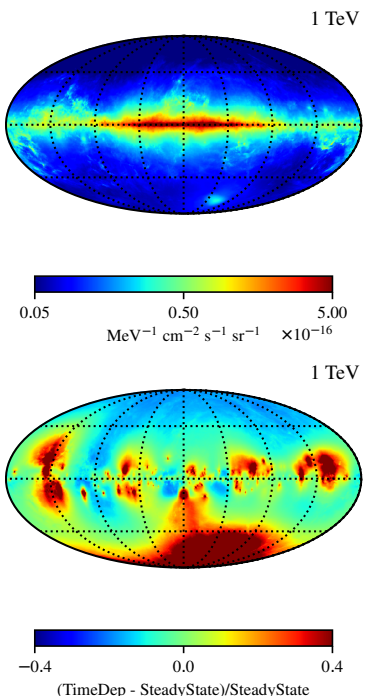

Figure 4: Total all-sky $\gamma$-rayintensity maps at selected energies for a GALPROP model including source discretization and time dependence (top) and corresponding fractional residual maps at same energies using the steady-state/continuous solution (bottom). From [12].

The previous work gives an indication of the delicacy of modeling the interstellar emission in the Fermi-LAT dataset, a significant background (or foreground) for most observations and especially critical for large-scale extended sources, when the standard distributed models, intended for the analysis of point sources, do not apply. [11] shows how a model can be constructed to be consistent with a collection of multiwavelength/multimessenger data, including radio, microwaves, $\gamma$ rays, and direct measurements of proton and electron spectra at Earth (AMS-02, PAMELA) and outside the solar sphere of influence (Voyager). In particular, updated magnetic fields based on synchrotron observations produce a more peaked Inverse Compton emission in the inner Galaxy with respect to the standard models used to analyze Fermi LAT data $(+30 \%$ at $10 \mathrm{GeV})$.

Temporal evolution is one feature that is consistently neglected in diffuse models, where the steady state solution is usually computed. Of course the main reason for this is the fact that adding time dependence to the sources and to cosmic ray propagation and (re-)acceleration is extremely resource intensive. In [12] the authors describe adding source discretization and time dependence to the GALPROP code, and how this affects results. Time evolution is simulated for $600 \mathrm{Myr}$, which is an order of magnitude longer than the dominant (propagation) time scale around a few $\mathrm{GeV}$, and the obtained model is compared with the equivalent continuous, steady-state version: see Fig. 3. While the overall structure appears unaffected, features appear like the bright spot at $1 \mathrm{TeV}$ near the south pole due to a nearby active source region, evident in the residual maps at all energies. The many novelties with respect to the usual steady-state/continuous models may provide clues to the origin of puzzling features in high-energy $\gamma$-ray data, such as the north/south asymmetry, and to a re-evaluation of the systematics affecting large-scale features in the $\gamma$-ray sky.

In [13] several results are reported for DAMPE observations. [14, 15] address a variety of 
topics pertaining the DAMPE detector operating as a $\gamma$-ray observatory. Here I focus on the search for dark matter annihilation lines [16], where the good energy resolution of the detector $(\sim 1 \%$ at $100 \mathrm{GeV}$ and above) plays a major role. For a search centered on the Galactic center and excluding the Galactic plane, spanning 3 years of data, the authors report upper limits that are competitive with Fermi-LAT results for almost twice the time.

\section{Extragalactic sources}

M31 is a complex system including the Andromeda galaxy and a large cosmic-ray and darkmatter halo, located very close to our Galaxy, covering a region in the sky of about $40^{\circ}$ in diameter. In [17], after selecting a tuning region embracing the region of interest and subtending approximately the same galactic location, the authors proceed as described in the previous section, analyzing 7.6 years of Fermi-LAT data until residuals are under control. Applying the derived diffuse emission model to the M31 region they report a 3-5\% residual around $10 \mathrm{GeV}$, which does not disappear when tweaking the interstellar emission model. The systematic uncertainties are significant, and a further dedicated work is announced.

In [18] the authors report the results of a search for periodicity over the lightcurves of 2276 Active Galactic Nuclei (AGN) detected with Fermi-LAT. Data above $1 \mathrm{GeV}$ are used to produce weekly lightcurves that are passed to 3 independent algorithms; a positive result on all 3 at $3 \sigma$ significance is required to claim detection. Out of the sample, 23 candidates are identified; in Fig. 4 an example is shown. This improves on the previous set of 5 candidate periodic signals.

Figure 5: Weekly lightcurve $(>1 \mathrm{GeV})$ and candidate periodic modulation for TXS 0518+211. From [18].

For 2 weeks in 2014 the blazar Mk501 was observed at TeV energies by the MAGIC and FACT Cherenkov telescopes in its highest state in 14 years. Analysis of the Fermi-LAT and MAGIC data in [19] suggests a feature in the spectrum around $3 \mathrm{TeV}$ at $2.9-4.5 \sigma$ significance. To explain such 
an unusually sharp feature one can suppose either a narrow electron distribution that somehow does not radiate itself away for some time, or a structured jet, or similar ad-hoc solutions. Remarkably, this feature does not reappear under very similar conditions a day later, but it remains unclear whether the feature disappeared or if the source is just powering down.

Among the AGNs blazars are the most energetic objects, and include some of the most energetic $\gamma$-ray sources. In [20] a model is proposed where the blazar emission is powered by the relativistic reconnection of magnetic field lines, accelerating particles into plasma blobs ("plasmoids") of various size. Remarkably, the model can reproduce several known features of this class of objects. The different dimension of the plasmoids translates into variation of the flux at different time scales, from minutes to weeks; the same population of plasmoids under different levels of the external radiation fields and under the effect of the relative illumination from big, bright ones on the fast, smaller ones naturally produce either Inverse-Compton dominance (FSRQs) or similar intensity of synchrotron Inverse-Compton peaks (BL Lac's).

Bright, high-redshift blazars are valuable cosmological probes, to study the AGN emission models and probe the level of extragalactic background light (EBL) along the line of sight. In [21] the authors suggest to search for flares from objects at $z>2.5$ for which the average emission is barely below the sensitivity limit of Fermi-LAT. A test statistics for detection in a time interval of one month is constructed, and evaluated on monthly time intervals for a sample of 100 undetected blazars. 21 monthly intervals for 7 sources have a detection, with chance probability of $5 \times 10^{-7} \%$, of these 4 are identified in the recently published 4FGL catalog, and 3 are completely new.

In [22] the amount of EBL absorption observed at $\mathrm{TeV}$ energies compared to the $\mathrm{GeV}$ spectrum is used to infer the redshift of blazar KUV 00311-1938, for which contradictory estimates exist.

[23] investigates the spectral properties of Extremely High Synchrotron Peak blazars, for which the spectral energy distribution peak at $\mathrm{TeV}$ energies (once EBL absorption is factored in). Using the 3rd and 4th Fermi-LAT catalogs and Cherenkov observations the authors find that the spectral characteristics are at tension with leptonic models, suggesting a hadronic scenario.

[24] describes the search for $\gamma$-ray counterparts for 76 Fast Radio Bursts and 108 FR0 compact radio galaxies in Fermi-LAT data. Sources are found with a pre-trial significance greater than $4 \sigma(2$ FRBs and 7 FR0s) but no correlations survive with high significance after considering the number of trials, time correlation (FRBs), and the impact of nearby sources.

Apart from the contribution from unresolved point-sources, the unresolved $\gamma$-ray background might contain a contribution from truly diffuse components (cosmic-ray interactions with the intergalactic medium, dark matter annihilation signals, ... ). In [25] the authors analyze Fermi-LAT data to derive constraints to the contributions of the unresolved source populations. They apply a generalized pixel-count statistics method, using specific phenomenological models for the emission of the resolved and unresolved $\gamma$-ray source populations, and in addition look at the angular correlations of the photon count distribution. The two methods provide complementary constraints, which will be investigated in more detail in the future.

The Fermi-LAT collaboration released the second catalog of Gamma-Ray Bursts (GRB) observed by the LAT, based on 10 years of data, with 186 events (17 short GRBs, 34 with known redshift); [26] presents a review. Even for a short review the amount of information is too much to summarize here, I will focus on two points: the temporal properties and the role of $>10 \mathrm{GeV}$ photons. In Fig. 4 the temporal properties of the low-energy and high-energy emission, measured 
by the Fermi-GBM and the Fermi-LAT detectors respectively, are shown. On the $x$ axis the characteristic times derived for the emission in the GBM band $\left(T_{X}\right.$ : time after trigger for which $X \%$ of the photons from the GRB were collected), on the $y$ axis the characteristic times for the emission in the LAT energy band ( $T_{L 0}$ and $T_{L 1}$ : times at which the first and last photons are collected). The three panels give some very generic properties of the dataset, in order left to right: the onset of the highenergy component happens after the onset of the low-energy component, the high-energy emission continues after the low-energy component has terminated, the high-energy emission starts before the low-energy emission is over (the outliers in orange correspond to events where the GRB was outside the LAT field of view at trigger time). Developing a coherent model featuring the first two properties is particularly difficult. A photon with energy above $50 \mathrm{GeV}$ was observed for less than $5 \%$ of the LAT GRBs, up to a maximum $95 \mathrm{GeV}$. Looking at the arrival time, such high-energy $\gamma$ rays arrive often after the lower-energy emission is over, with no other particular trend, either immediately after or after a rather long time (100's of seconds); again this is particularly challenging to model.

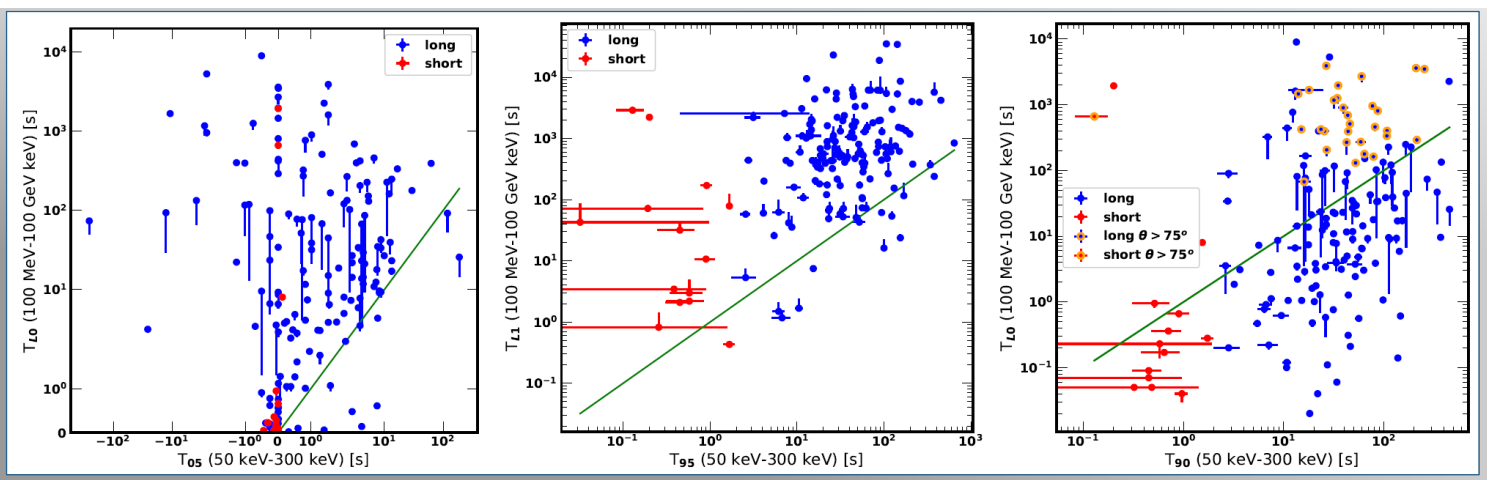

Figure 6: Temporal properties of LAT and GRB emission in the Second Fermi-LAT GRB Catalog, see text for details. From [26].

The importance of such rare high-energy photons from GRBs lead to the effort to recover some more. In [27] a dedicated analysis is used to identify photons coming from known LAT GRBs which do not leave a signature in the tracker, but only in the calorimeter of the LAT. The challenge is to ensure a good angular resolution and a sufficient level of background rejection for these peculiar events. By restricting to energies $>10 \mathrm{GeV}$, where the statistics is low, and to a sample of time intervals around 24 GRBs, a successful analysis returns 4 new events. As an example, for GRB090926A one new photon at $50 \mathrm{GeV}$ is found (observed energy, corresponding to $157 \mathrm{GeV}$ in the GRB frame of reference). This arrived $424 \mathrm{~s}$ after the trigger, quite a challenge to the current synchrotron emission models. To have a feeling of the low statistics in this energy regime we observe that although the point-spread function is $1.7^{\circ}(\sim 30 \times$ worse than events with the same energy in the standard event classes) and that the event arrives within a $>400 \mathrm{~s}$ time window, the chance probability of this being a background event is $\sim 0.02 \%$.

Many classes of models predict some amount of polarization to be detected in the GRB emission. POLAR is a dedicated plastic-scintillator instrument that operated on the Chinese space laboratory Tiangong-2 for 6 months, detecting 55 GRBs in the Compton regime (at 50-500 keV)[28]. From this sample, 5 GRBs were selected, based on their optimal observing condition, for an analy- 
sis of their polarization. The average polarization results $(\sim 10 \%)$ are lower than what is predicted by some models. Unfortunately a breakdown of the power supply unit terminated this experiment prematurely. An improved version, POLAR-2, is under development and is expected to fly in 2024, details are given in [29].

CALET on the International Space Station has been in operation since 2015, monitoring the $\gamma$-ray sky from $1 \mathrm{GeV}$ to $10 \mathrm{TeV}$ with a wide field-of-view. In $[30,31]$ the authors report on the progress in event analysis, and describe the search for transient events. Once more the limited photon fluxes in this high-energy regime implies that one can look for pairs of events within the angular resolution element to signal a transient; the detection of an AGN flare validates the technique. The search is of particular interest for GRBs as counterparts of gravitational wave (GW) events, given the wide field of view that can cover a great part of the localization uncertainties of GW detectors; an overview of the automated search system is provided and the current upper limits for past events are discussed, see also Fig.4. In addition to the main instrument, a GRB detector (CGRB) is on board, sensitive in the energy range from $7 \mathrm{keV}$ to $20 \mathrm{MeV}$; details about 4 short GRBs out of the 161 observed are given in [32].

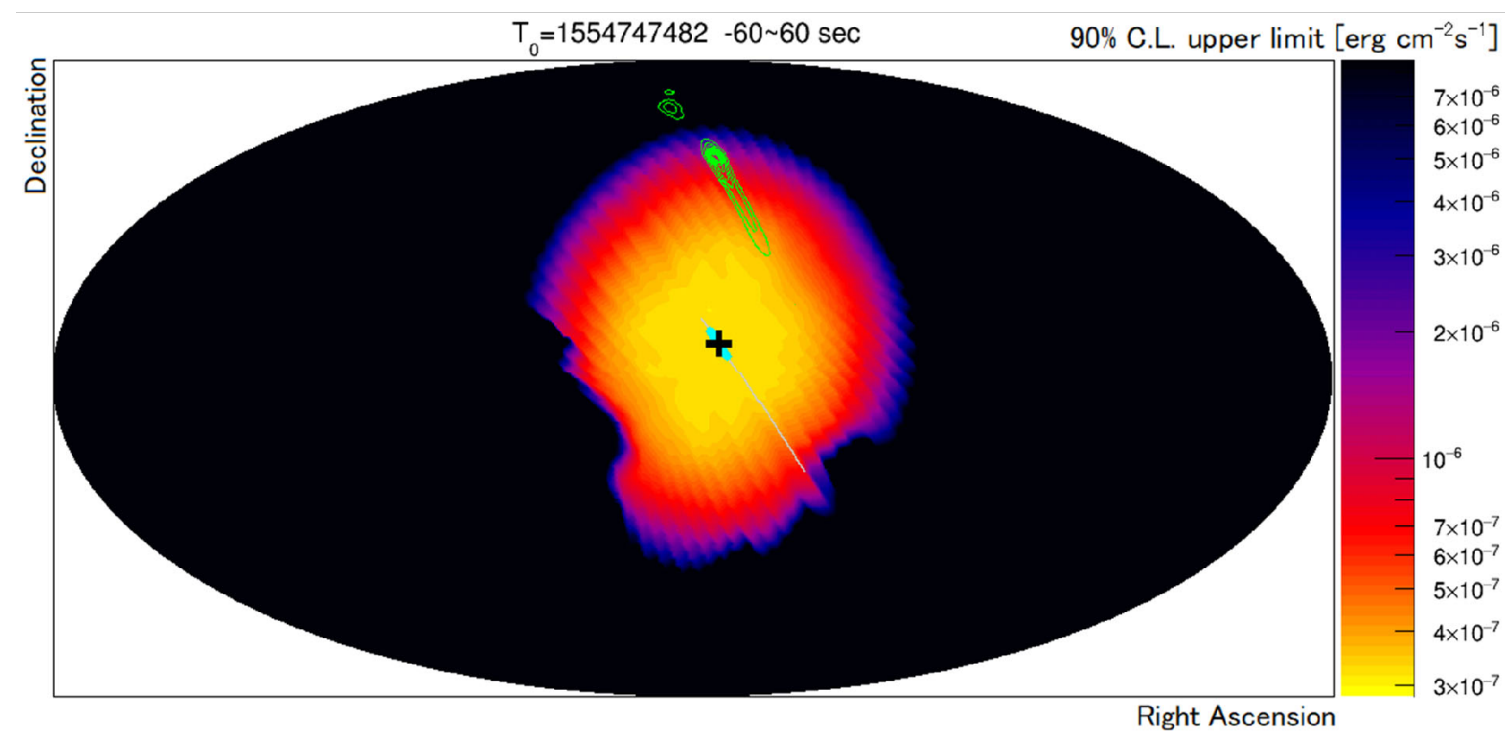

Figure 7: $90 \%$ confidence level upper limits on $\gamma$-ray emission from the GW event S190408an in the 1$10 \mathrm{GeV}$ band with CALET; in green the localization contours reported by LIGO/Virgo. From [30].

Several empirical correlations have been proposed to infer the distance of a GRB from $\gamma$-ray observations, based on prompt and afterglow observations. In [33] the authors compare the widely used Amati relation with a relation derived assuming a multi-component prompt emission spectral scenario. Testing this with a sample of 3 bright bursts with known redshift they found a good agreement between the two estimates, while for 3 fainter ones with no redshift information some discrepancy is observed, with smaller values favored by the Amati relationship.

POSyTIVE, the POpulation Synthesis Theory Integrated project for very high-energy emission, is a Cherenkov Telescope Array project to make realistic predictions for the observation of GRBs [34]. The mock GRB population used by POSyTIVE is calibrated using the 40-year dataset of multi-wavelength GRB data, and simulated observations are performed with the publicly avail- 
able CTA software tools.

\section{Updates and new missions}

The energy range between the bands covered by the hard X-ray observatories and FermiLAT, i.e. from where Compton scattering is the prevailing interaction mechanism up to where the cross sections for pair-production and Compton interaction compete, is severely under-explored. Currently SPI on board the INTEGRAL mission covers the range $18 \mathrm{keV}-8 \mathrm{MeV}$ with good performance and a field of view of tens of degrees since 2002. The situation is starting to change, with COSI (balloon flight) and POLAR (see §4); for both of these an upgrade is planned. The last fullsky observatory, COMPTEL on board the Compton Gamma-Ray Observatory (CGRO), provided us with many a lesson about the complexity of observations in the Compton regime, especially about dealing with backgrounds: external, and internal due to material activation. AMEGO, the All-Sky Medium Energy Gamma-ray Observatory [35] is a proposed NASA probe-class mission, to investigate the energy range from $200 \mathrm{keV}$ to $>10 \mathrm{GeV}$ with sensitivity a factor of 20-50 better than previous instruments. The two interaction processes require different approaches in both detection and data analysis, and consequently in the instrument concept. AMEGO addresses this with a Silicon tracker with no converter foils, to reduce the scattering of secondaries, and with two calorimeter subsystems, each optimized for maximum performance in dealing with Compton and pair-production events, respectively. The expected scientific output is way too vast to enumerate here. Let it suffice to say that AMEGO's discovery potential derives from the combination of high continuum sensitivity and of the capability for nuclear line spectroscopy and $\gamma$-ray polarization; for a graphical summary see Fig. 5. A review of topics related to the study of cosmic rays and of the interstellar medium is given in [36]. The current status of the prototype subsystems is described in [37].

Another proposed $\mathrm{MeV}$ instrument with similar scientific objective but a slightly different design and mission plan is AllSky-ASTROGAM [38]. Notably, AS-ASTROGAM is designed to operate from the Sun-Earth second Lagrange point and achieve an almost complete coverage of the sky at all times.

Several contributions featured new proposals for next-generation instruments operating at $\sim$ $\mathrm{MeV}$ energies, a confirmation of the interest towards the wealth of information hiding in this energy band. GalacticCenterExplorer is a new mission concept for a coded-mask imaging calorimeter to survey the Galactic center with high energy and angular resolution [39]. MAMBO is a BGO spectrometer dimensioned to fit into a 6-liter nano-satellite, to measure the $\mathrm{MeV}$ extragalactic $\gamma$ ray background between $\sim 300 \mathrm{keV}$ to $\sim 30 \mathrm{MeV}[40]$.

GRAINE is a $\gamma$-ray observatory based on a stack of nuclear emulsion films [41]; demonstrative balloon flights were performed in 2011, 2015 and 2018 with different apertures, and a new flight with a much increased acceptance is proposed for 2021. The excellent intrinsic position accuracy of nuclear emulsions $(\sim 50 \mu \mathrm{m})$ allows precise tracking of the $\mathrm{e}^{ \pm}$pairs, giving very good angular resolution $\left(\sim 0.1^{\circ}\right.$ at $\left.100 \mathrm{GeV}\right)$ and polarization sensitivity. There can be no real-time data acquisition (the stack needs to be recovered and analyzed) but during the flight the instrument is dead-time free, and timing information can be encoded into one film (at bottom) used as time-stamper, shifting at constant speed for the entire mission time to misaling passing tracks by an amount proportional to 
Figure 8: Scientific capabilities of the AMEGO observatory; from [35].

time. The flight in 2018 was dedicated to the observation of Vela, and data analysis is approaching completion [42]. The flight in $\sim 2022$ will have various targets: Galactic center, the W44 SNR, transients, etc.

At lower energies, detectors of X-ray polarimetry are also being proposed, e.g. Gas Electron Multipliers (GEMs) on IXPE. The simulation of a GEM detector is described in [43], to characterize the detector response as a function of the operational parameters (pressure, voltage, temperature).

Burstcube is a 6-liter nano-satellite for the observation of GRBs, currently in its development and testing phase to prepare for launch readiness in 2021; see [44] and Fig. 5. Despite the small volume the performance approaches that of the Fermi-GBM, increasing the sky coverage of existing facilities for a small fraction of the cost of a larger mission. Currently, between SWIFT-BAT and Fermi-GBM $60 \%$ to $85 \%$ of the sky is under observation most of the time; the addition of another instrument with comparable performance will bring this figure to $>80 \%$ of the sky most of the time, with the whole sky being covered $\sim 20 \%$ of the time.

\section{References}

[1] K.C.Y. Ng, The surprising gamma ray emission from the Sun, in proceedings of 36th International Cosmic Ray Conference, PoS ( ICRC2019) 588.

[2] S. Manconi et al., Detection of a gamma-ray halo around Geminga with the Fermi-LAT and 

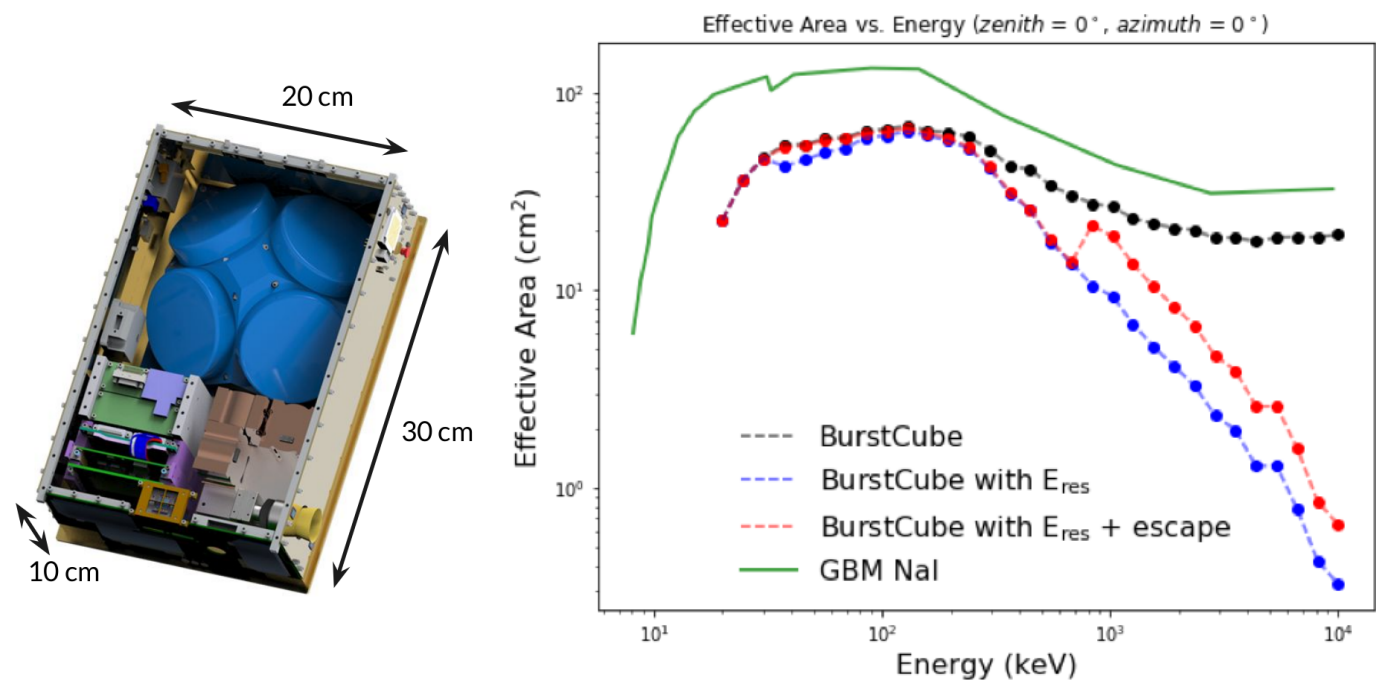

Figure 9: Left: the BurstCube satellite; right: effective area versus energy of BurstCube, compared to the Fermi-GBM. From [44].

implications for the positron flux, in proceedings of 36th International Cosmic Ray Conference, POS (ICRC2019) 580.

[3] Q. Kaplan et al., Results form the long-term Fermi-LAT observations of the white dwarf binary pulsar AR Scorpii, in proceedings of 36th International Cosmic Ray Conference, POS (ICRC2019) 602.

[4] R. Walter et al., $\eta$ Carinae: particle acceleration and multi-messenger aspects, in proceedings of 36th International Cosmic Ray Conference, POS (ICRC2019) 609.

[5] G. Principe et al., Energy-dependent morphology of the pulsar wind nebula HESS J1825-137 seen by the Fermi-LAT, in proceedings of 36th International Cosmic Ray Conference, POS (ICRC2019) 595.

[6] F. De Palma et al., Shell like supernova remnants observed with Fermi-LAT, in proceedings of 36th International Cosmic Ray Conference, POS (ICRC2019) 560.

[7] D. Green et al., Gamma-ray spectral and morphological study of HESS J1912+101 observed by MAGIC and Fermi-LAT, in proceedings of 36th International Cosmic Ray Conference, POS (ICRC2019) 564 .

[8] A. Mares et al., Efficient particle acceleration from HESS J1640-465 and the PeVatron candidate HESS J1641-463, in proceedings of 36th International Cosmic Ray Conference, POS (ICRC2019) 582.

[9] M. Pohl et al., Particle acceleration at, and gamma-ray emission from supernova remnants in chemically enriched media, in proceedings of 36th International Cosmic Ray Conference, POS (ICRC2019) 592.

[10] T. Joubaud et al., Cosmic rays in the Orion-Eridanus superbubble, in proceedings of 36 th International Cosmic Ray Conference, POS (ICRC2019) 568.

[11] E. Orlando, A consistent model of the interstellar gamma-ray emission to interpret Fermi LAT observations of diffuse emissions, in proceedings of 36th International Cosmic Ray Conference, POS (ICRC2019) 589. 
[12] T. A. Porter et al., High-energy gamma rays from the Milky Way: time-dependent modelling of cosmic ray propagation and non-thermal emissions with the GALPROP code, in proceedings of 36th International Cosmic Ray Conference, POS ( ICRC2019) 594.

[13] X. Li et al., Recent gamma-ray results from DAMPE, in proceedings of 36th International Cosmic Ray Conference, PoS ( ICRC2019) 576.

[14] M. Muñoz et al., Gamma-ray pulsars with DAMPE, in proceedings of 36th International Cosmic Ray Conference, POS ( ICRC2019) 587.

[15] W. Jiang et al., Boresight alignment with the DArk Matter Particle Explorer, in proceedings of 36th International Cosmic Ray Conference, POS ( ICRC2019) 567.

[16] Z. Shen et al., Search for a gamma-ray line feature with DAMPE, in proceedings of 36th International Cosmic Ray Conference, PoS ( ICRC2019) 601.

[17] C. Karwin et al., Fermi-LAT observations of gamma-ray emission towards the outer halo of M31, in proceedings of 36th International Cosmic Ray Conference, PoS ( ICRC2019) 570.

[18] P. Peñil, Systematic search for gamma-ray periodicity in Fermi-LAT blazars, in proceedings of 36th International Cosmic Ray Conference, POS ( ICRC2019) 591.

[19] J. Becerra Gonzalez et al., Study of the variable broadband emission of Markarian 501 during the most extreme Swift X-ray activity, in proceedings of 36th International Cosmic Ray Conference, POS (ICRC2019) 554.

[20] I. Christie et al., Radiative signatures of relativistic reconnection in blazar jets, in proceedings of 36th International Cosmic Ray Conference, POS ( ICRC2019) 559.

[21] M. Kreter et al., Search for high-redshift blazars with Fermi/LAT, in proceedings of 36th International Cosmic Ray Conference, POS ( ICRC2019) 573.

[22] M. Fernandez Alonso et al., Redshift of the Blazar KUV 00311-1938: Modeling the EBL Absorption, in proceedings of 36th International Cosmic Ray Conference, POS ( ICRC2019) 563.

[23] K. K. Singh et al., Intrinsic GeV-TeV gamma-ray emission from EHSP blazars, in proceedings of 36th International Cosmic Ray Conference, POS ( ICRC2019) 603.

[24] S. Best and J. L. Bazo Alba, Search for gamma-ray counterparts of newly discovered radio astrophysical sources, in proceedings of 36th International Cosmic Ray Conference, PoS ( ICRC2019) 553.

[25] F. Donato et al., Probing the gamma-ray source populations with photon count statistics and anisotropies, in proceedings of 36th International Cosmic Ray Conference, POS ( ICRC2019) 581.

[26] E. Bissaldi, High-energy emission from GRBs: 10 years with Fermi-LAT, in proceedings of 36th International Cosmic Ray Conference, POS ( ICRC2019) 555.

[27] M. Takahashi et al., Very-high-energy GRB events in novel Fermi-LAT photon data and their emission mechanism, in proceedings of 36th International Cosmic Ray Conference, P OS ( ICRC2019) 606.

[28] J Sun et al., Overview of the POLAR mission, in proceedings of 36th International Cosmic Ray Conference, POS ( ICRC2019) 605.

[29] M. Kole et al., POLAR-2: the first large scale gamma-ray polarimeter, in proceedings of 36th International Cosmic Ray Conference, POS ( ICRC2019) 572. 
[30] M. Mori et al., High-energy gamma-ray observations using the CALorimetric Electron Telescope (CALET) on the ISS, in proceedings of 36th International Cosmic Ray Conference, POS (ICRC2019) 586.

[31] N. W. Cannady, CALET upper limits on GeV-energy gamma-ray burst emission, in proceedings of 36th International Cosmic Ray Conference, POS ( ICRC2019) 557.

[32] Y. Kawakubo, CALET upper limits on GeV-energy gamma-ray burst emission, in proceedings of 36th International Cosmic Ray Conference, POS ( ICRC2019) 571.

[33] J. R. Sacahui et al., Constraining GRBs pseudo-redshifts using different empirical correlations, in proceedings of 36th International Cosmic Ray Conference, POS ( ICRC2019) 597.

[34] M. G. Bernardini et al., POSyTIVE - a GRB population study for the Cherenkov Telescope Array, in proceedings of 36th International Cosmic Ray Conference, POS ( ICRC2019) 598.

[35] A. Moiseev, All-Sky Medium Energy Gamma-ray Observatory (AMEGO), in proceedings of 36th International Cosmic Ray Conference, POS ( ICRC2019) 583.

[36] E. Orlando et al., Cosmic rays and the interstellar medium with the All-Sky Medium Energy Gamma-ray Observatory (AMEGO), in proceedings of 36th International Cosmic Ray Conference, POS (ICRC2019) 590

[37] S. Griffin, Subsystem development for the All-Sky Medium Energy Gamma-ray Observatory (AMEGO) prototype, in proceedings of 36th International Cosmic Ray Conference, POS (ICRC2019) 565.

[38] M. Mallamaci et al., All-Sky-ASTROGAM: a MeV Companion for Multimessenger Astrophysics, in proceedings of 36th International Cosmic Ray Conference, POS ( ICRC2019) 579.

[39] A. Moiseev et al., New mission concept: investigation of the Galactic Center with GalacticCenterExplorer (GalCenEx), in proceedings of 36th International Cosmic Ray Conference, POS (ICRC2019) 585.

[40] T. Verstrand et al., The Micro Astrophysical MeV Background Observatory (MAMBO), in proceedings of 36th International Cosmic Ray Conference, PoS ( ICRC2019) 608.

[41] S. Takahashi, GRAINE project and first results on 2018 balloon-borne experiment, in proceedings of 36th International Cosmic Ray Conference, POS ( ICRC2019) 607.

[42] H. Rokujo et al., Gamma-ray imaging performance of nuclear emulsion telescope in GRAINE-2018 balloon experiment, in proceedings of 36th International Cosmic Ray Conference, POS (ICRC2019) 596.

[43] A. Jung, Simulating an X-ray polarimeter gas pixel detector, in proceedings of 36th International Cosmic Ray Conference, PoS ( ICRC2019) 569.

[44] J. R. Smith et al., BurstCube: concept, performance, and status, in proceedings of 36th International Cosmic Ray Conference, PoS ( ICRC2019) 604. 